\title{
El Portafolio como recurso para la reflexión y la autoevaluación en alumnos con dificultades de aprendizaje
}

\author{
Andrés Canga Alonso \\ Universidad de la Rioja \\ Recibido: 12 septiembre 2010 / Aceptado 12 diciembre 2010 \\ ISSN: $1697-7467$
}

\begin{abstract}
RESUMEN: Este artículo analiza la inclusión del Portafolio Europeo de las Lenguas en el curriculum del Programa de Diversificación Curricular en un centro educativo de Asturias (España) para fomentar la reflexión y la autoevaluación en el proceso de aprendizaje de una lengua extranjera. En primer lugar, se relaciona el Marco Común Europeo de Referencia para las Lenguas y el PEL con la autonomía de los aprendices. Posteriormente, se examinan las características del grupo de informantes y las actividades realizadas. Los resultados ponen de manifiesto que el PEL es una herramienta adecuada para favorecer la reflexión en alumnos con dificultades de aprendizaje.

Palabras clave: reflexión, portafolio y autoevaluación.
\end{abstract}

The Portfolio as a resource for reflection and self-evalaution with students having learning difficulties

\begin{abstract}
This paper shows how the European Language Portfolio has been integrated into the Diversificación Curricular English syllabus at a Secondary School in Asturias (Spain) to promote self-reflection and self-assessment. It starts with an explanation of the relationship between the Common European Framework, the ELP, and learner autonomy. The second part deals with the main characteristics of the students, as well the tasks they have done during the study. The findings show that the ELP is a useful tool to promote self-assessment and self-reflection in students with learning difficulties.
\end{abstract}

Keywords: self-reflection, portfolio and self-assessment.

\section{INTRODUCCIÓN}

En la última década, el Marco Común Europeo de Referencia para las Lenguas $(M C E R L)$ se ha convertido en una referencia para todos los estamentos implicados en el aprendizaje de lenguas. Tiene como objetivo establecer una política educativa que, tal como señala Dobson (2006), no solo fomente el aprendizaje de lenguas sino también ayude a los aprendices a entender los aspectos culturales asociados al idioma que aprenden para así poder establecer vínculos entre estructuras gramaticales y vocabulario que hubiesen adquirido previamente (competencia plurilingüe). 
$\mathrm{Su}$ influencia es tal que el Consejo de Ministros del Consejo de Europa en su reunión del día 2 de julio de 2008 (http://www.coe.int/t/dg4/linguistic/) aprobó la Recomendación $\mathrm{CM} / \operatorname{Rec}(2008) 7$ para potenciar su uso en los estados miembros con el objetivo de promover y facilitar la cooperación entre las instituciones educativas de los países miembros. El MCERL propone un enfoque centrado en el alumno, basado en la acción y el aprendizaje por competencias, tomando en cuenta la dimensión social y cultural de cada lengua de modo global de tal manera que le permita establecer relaciones entre las lenguas que aprende, es decir, desarrolle su competencia plurilingüe. Este modelo de aprendizaje fomenta el aprendizaje a lo largo de la vida y, por tanto, la autonomía de los aprendices ya que los estudiantes se hacen responsables de su proceso de aprendizaje (Holec, 1981; Little, 2007), van modelando su forma de aprender de modo progresivo (Vygotsky, 1978; Little, 2007) y se vuelven individuos más reflexivos. Para favorecer este proceso, el profesor debe tener en cuenta las capacidades y necesidades del alumnado que tiene a su cargo y prepararlos lo mejor posible para responder a las necesidades que plantea el mundo globalizado en el que nos ha tocado vivir.

Por otro lado, el MCERL describe las actividades que el alumno debe ser capaz de llevar a cabo para cada una de las destrezas (leer, escribir, escuchar, hablar y conversar) que emplea en cada una de las lenguas que aprende, permitiéndole saber, en todo momento, en qué nivel se encuentra de los seis reconocidos (A1-C2).

Por último, este documento pone énfasis en el aprendizaje social e interactivo que implica tres principios:

a) Learner empowerment by transferring part of the responsibility for teachinglearning to pupils by giving them different options and support.

b) Learner reflection to make students think about what they are learning and why it is necessary for them.

c) Appropriate target language use by using the foreign language for genuine communicative purposes (Little, Ridley y Ushioda, 2002; Little, 2003 y 2007).

Con el objetivo de poner en práctica tanto las recomendaciones del Consejo de Ministros del Consejo de Europa como las indicaciones recogidas en el MCERL, desde el Consejo de Europa se ha impulsado el uso del Portafolio Europeo de las Lenguas (PEL). EL PEL ha sido diseñado para cubrir una doble función: informativa y pedagógica y comprende tres partes: pasaporte, biografía lingüística y dossier (Dobson 2006: 28-29). El estudio al que alude este artículo, que se expondrá en detalle en el apartado sobre metodología, se centró en la función pedagógica, puesto que favorece la reflexión sobre el proceso de aprendizaje a través de los descriptores que se presentan para cada una de las destrezas y con ello fomenta el desarrollo de la autonomía de los discentes (Cassany, 2006; Little, Ridley y Ushioda, 2002; Palacios, 2006). Para favorecer dicho proceso se utilizaron los descriptores de la biografía lingüística y el dossier donde los alumnos recogieron aquellos documentos que consideraron más relevantes para su aprendizaje. A pesar de ello, al comienzo del estudio, se cumplimentaron algunos de los apartados del pasaporte para que los alumnos fuesen conscientes de su importancia a lo largo de su trayectoria educativa y profesional.

En España, existen cuatro portafolios validados por el Consejo de Europa: Educación Infantil (3-7) Educación Primaria (8-12), Secundaria (12-18) y adultos. El presente artículo 
se centrará en el portafolio de Secundaria dado que los alumnos forman parte del Programa de Diversificación Curricular en los dos últimos años de la enseñanza obligatoria $\left(3^{\circ}\right.$ y $4^{\circ}$ de ESO).

\section{Metodología}

\subsection{Objetivos}

Teniendo en cuenta que el Consejo de Europa se propone para este comienzo de siglo el desarrollo del pluriligüismo a través de un proceso autónomo de aprendizaje a lo largo de la vida, el presente estudio se planteó con un doble objetivo:

a) Favorecer la autonomía de los discentes haciéndoles responsables de su propio aprendizaje a través de la reflexión y la autoevaluación en sus portafolios.

b) Comprobar si el alumnado alcanza el nivel $\mathrm{A} 1^{+}-\mathrm{A} 2$ de acuerdo con el $M C E R^{1}$ a partir de las actividades realizadas durante el periodo lectivo y su reflexión posterior en el portafolio.

\subsection{Participantes instrumentos y procedimientos}

Los informantes del presente estudio son trece alumnos (once chicos y dos chicas) pertenecientes al Programa de Diversificación Curricular de un centro educativo de Oviedo (Asturias). Los Programas de Diversificación Curricular están formados por estudiantes que presentan dificultades de aprendizaje (DA) que, por lo general, vienen acompañadas de falta de motivación hacia el aprendizaje derivada de los fracasos académicos que han ido acumulando a lo largo de su trayectoria educativa. Por estas razones, el currículum debe ser adaptado de modo que puedan alcanzar las competencias básicas que les permitan obtener el título de graduado en Educación Secundaria Obligatoria. Este tipo de programas se desarrollan durante dos años académicos ( $3^{\circ}$ y $4^{\circ}$ de ESO) aunque es posible incluir alumnos en el segundo año del programa en función de sus características y necesidades.

Se emplea un metodología activa y participativa con el objetivo de que los alumnos vayan recuperando la autoestima y con ello su motivación aumente, por lo que trabajan en grupos de entre ocho y quince alumnos en tres áreas curriculares: ámbito sociolingüístico (Lengua Castellana y Literatura y Ciencias Sociales Geografía e Historia), ámbito científico-tecnológico (Matemáticas, Biología y Física y Química) y lengua extranjera (Inglés).

La lengua extranjera debe ser impartida siguiendo un enfoque comunicativo para que los alumnos pertenecientes al programa adquieran las competencias básicas de acuerdo

${ }_{1}$ Tal como se manifiesta en la página web del OAPEE: «El usuario básico: A1 y A2 es capaz de comunicarse, en situaciones muy cotidianas, con expresiones de uso muy frecuente y utilizando vocabulario y gramática básica.» (http://www.oapee.es/inicio/iniciativas/portfolio/niveles-de-competencia.html, visitada el 11 de febrero de 2010). 
con las directrices que aparecen en el MCERL, considerándose positivo si logran alcanzar el nivel $\mathrm{A}^{+}$al final del programa. El resto de las asignaturas que forman parte del currículum de $3^{\circ}$ y $4^{\circ}$ de ESO las cursan con el resto de compañeros que no presentan DA no pudiendo superar los treinta alumnos por grupo.

Para preservar la identidad de los informantes, se utilizará un número del 1-13 precedido de la letra e (estudiante). Todos los informantes presentan falta de motivación hacia el aprendizaje debido a su fracaso escolar y esta desmotivación es aún mayor hacia la lengua extranjera, puesto que como ellos reconocen al comienzo del primer curso del programa es una asignatura inútil que no les aporta nada a su formación. Además, cinco informantes (e1, e2, e3, e4 y e12) presentan serias DA con respecto a comprensión y expresión escrita. Cuatro de ellos (e5, e6, e9 y e11) han sido expulsados del centro escolar durante algún periodo a lo largo de su trayectoria académica debido a faltas de conducta graves contra las normas de convivencia. Por último, hay tres alumnos (e5, e9 y e11) que tienen problemas psicológicos que afectan a su interacción con los miembros de la comunidad educativa. Los estudiantes restantes (e7, e8, e10 y e13) no presentan ningún problema añadido a su falta de motivación.

Siguiendo las directrices marcadas para los Programas de Diversificación, el estudio se llevó a cabo durante dos cursos académicos (2006-2007 y 2007-2008). Todos los alumnos, salvo e12 y e13, permanecieron dos años en el programa, por lo que todos serán tenidos en cuenta a la hora de analizar sus resultados, a pesar de que e5 abandonó sus estudios al cumplir la mayoría de edad y e6 fue expulsado a finales de mayo de 2008 por cometer una falta muy grave contra las normas de convivencia del centro.

En lo que respecta a los instrumentos utilizados para medir los objetivos antes citados, en primer lugar se pidió a los alumnos que llevasen un diario de aprendizaje en el que reflejasen su reflexiones sobre las actividades realizadas en el aula de lengua inglesa, puesto que los diarios de aprendizaje, permiten a los alumnos sentirse libres para expresar sus opiniones sobre el proceso de aprendizaje, hacerse más reflexivos e incluso puede ayudarles a mejorar su capacidad de escritura. Por estas razones, se convierten en una herramienta necesaria para facilitar la autonomía del aprendiz en cuanto a reflexión, toma de conciencia y control del propio aprendizaje (Wallace 1998). Dadas las peculiaridades del grupo analizado, se permitió que los diarios pudieran ser elaborados tanto en la lengua materna como en lengua inglesa durante la última parte de la sesión en el aula de informática.

Dado que el Programa de Diversificación Curricular tiene asignadas tres horas lectivas durante el primer año y dos durante el segundo, se optó por utilizar el aula de informática una vez a la semana durante el primer año del programa y una vez cada dos semanas durante el segundo año. En esta sesión los estudiantes debían reforzar los puntos gramaticales abordados en el aula tomando como referencia las páginas web sugeridas por el profesor a comienzos del curso (www.isabelperez.com, http://clicknlearn.net y www.english-hilfen.de). Desde el segundo trimestre del primer año del programa hasta el final del estudio, los alumnos realizaron trabajos individuales y en grupos sobre temas de su interés (intereses y aficiones, moda, deporte, música...) debiendo seleccionar y resumir la información para presentarla a sus compañeros y plasmarla en una cartulina para colocar en el aula. El objetivo de estas actividades es que el alumno trabaje las cinco destrezas que aparecen reflejadas en el portafolio (leer, escribir, escuchar, hablar y con- 
versar), puesto que los estudiantes debían presentar la información a sus compañeros (escribir, leer y hablar), y prestar atención a la exposición, para, así, poder responder adecuadamente a una serie de cuestiones planteadas por los autores del trabajo que, posteriormente, eran evaluadas por el profesor.

Al final de cada actividad, los informantes debían reflexionar sobre lo aprendido en sus portafolios partiendo de sus trabajos individuales y grupales contrastándolos con los descriptores para los niveles A1 y A2 de la biografía lingüística y marcando las casillas que mejor describían su competencia en inglés. El profesor revisaba cada portafolio individualmente con los alumnos para que le justificasen verbalmente el porqué de su elección y una vez se habían hecho varias reflexiones se hacía una puesta en común con todos los alumnos para valorar su proceso de aprendizaje. Por ultimo, cada estudiante guardaba una copia de las actividades en el dossier y anotaba la fecha y la razón por la que decidía guardar el documento.

Una vez al trimestre, tomando como referencia las actividades propuestas por Cassany (2006), Little y Perclová (2002) y en la Guía del Portfolio Europeo de las Lenguas para Enseñanza Secundaria (MEC), los discentes realizaron diversas actividades en la biografía lingüística de sus portafolios sobre las lenguas que conocían y los contextos en que empleaban cada una de ellas. Como actividad final debieron elaborar su abanico lingüístico empleando todas las lenguas en las que creían ser capaces de comunicarse.

Durante el segundo año, los participantes establecieron contacto con estudiantes de español de un centro privado del Reino Unido a través del e-mail tándem. Esta metodología de aprendizaje desarrollada en la década de los 90 por investigadores como Brammerts, Calvert y Little se fundamenta en dos principios que están estrechamente ligados a la autonomía de los aprendices y el aprendizaje a lo largo de la vida: reciprocidad y autonomía. Por reciprocidad se entiende: «each partner brings certain skills and abilities which the other partner seeks to acquire and in which both partners support each other in their learning in such a way that both benefit as much as possible from their working together» (Brammerts, 2003: 29). Autonomía implica que ambos aprendices son responsables de su proceso de aprendizaje, así que ellos mismos deciden qué quieren aprender, cómo y cuando quieren aprenderlo y que ayudan precisan de su pareja tándem para lograr sus objetivos (Brammerts, 2003: 29). Los alumnos deben utilizar la lengua materna y la lengua extranjera en cada una de sus comunicaciones debiendo de corregir los errores de su pareja siguiendo las pautas de corrección que se les entregan al principio de curso. Dadas las características del grupo y las diferencias de nivel existentes entre sus miembros se optó porque trabajasen en tríos formados por dos alumnos españoles y uno inglés, salvo en el caso de e6 y e11 que trabajaban individualmente.

La actividad se realizaba cada dos semanas abordando temas relacionados con las aficiones y gustos, las formas de celebrar las fiestas más tradicionales en ambos países (navidad, semana santa, carnaval, fiestas locales y regionales), comparación entre ambos colegios y sistemas educativos y hábitos saludables. Por medio de estas actividades se establece una comunicación intercultural entre los participantes (Stickler y Lewis, 2003: 97-98), aspecto de vital importancia para el Consejo de Europa y que aparece de modo reiterativo en el MCERL, tal como se expuso en el primer apartado de este artículo, puesto que el aprendizaje de una lengua es un proceso activo en el que los aprendices no sólo deben adquirir las competencias fonéticas y gramaticales de la lengua meta, sino también 
ser conscientes del modo en el que viven y se comportan las comunidades de hablantes de la lengua que aprende. El desarrollo de esta competencia denominada competencia comunicativa intercultural (Byram, 1997) ha de ser básica para favorecer la reflexión y autoevaluación del propio proceso de aprendizaje. Por ello, tal como se mostrará en el epígrafe siguiente, una vez recibida la respuesta al correo electrónico enviado se pedía a los informantes que hiciesen una breve reflexión sobre los aspectos más significativos para su aprendizaje desde el punto de vista lingüístico y cultural.

En cuanto a la introducción de las estrategias de aprendizaje como elemento de reflexión y autoevaluación, se adaptaron varias de las actividades propuestas por Palacios Martínez (2006: 129-170) referidas a autoevaluación y estrategias de lectura y comprensión de textos. Además, se profundizó en esta reflexión con el apartado sobre $M i$ Manera de Aprender de la biografía lingüística del portafolio.

Por último, durante el primer año del estudio se pidió a todos los participantes que cumplimentasen un cuestionario al final de cada trimestre, en el que se les preguntaba no sólo sobre las calificaciones que esperaban obtener en su evaluación trimestral, sino también se les requería que fijasen objetivos de mejora en su aprendizaje para el siguiente trimestre. Desde diciembre de 2007, dadas las peculiaridades del grupo antes mencionadas, se decidió de mutuo acuerdo con los alumnos que la reflexión se llevaría a cabo en el diario para evitar tener que cumplimentar tantos cuestionarios, puesto que sus tutores también les pedían una autoevaluación de todas las materias al final de cada trimestre. Al final del primer curso académico (junio de 2007) se les administraron dos cuestionarios, tomados de la memoria de experimentación del portafolio en España (http://www.oapee.es/ documentum/MECPRO/Web/weboapee/iniciativas/portfolio/portfolios-validados-esp/secun$\mathrm{daria} /$ castellano/memoriaexperimentacionsecundaria.pdf?documentId=0901e72b800044b2, visitada el 11 de febrero de 2010) sobre la importancia del portafolio en su proceso de aprendizaje de la lengua extranjera para contrastar sus impresiones con las que tenía el docente y verificar, como así se detallará en el apartado siguiente, si el alumnado estaba de acuerdo con esta dinámica de trabajo o por el contrario sugerían algún tipo de cambios.

\section{Resultados}

Para analizar los resultados obtenidos por los informantes se hará una referencia a cada uno de los instrumentos analizados en el epígrafe precedente añadiendo al análisis un ejemplo del trabajo realizado por los participantes.

En primer lugar, los diarios de aprendizaje han sido una herramienta adecuada y útil para que los discentes fueran conscientes de su proceso de aprendizaje de un modo reflexivo, puesto que, como se pone de manifiesto en el extracto del diario de e8 que se muestra a continuación, los estudiantes son más reflexivos al final del estudio que al comienzo del mismo y valoran positivamente la experiencia de aprendizaje:

Estuve en la página clicknlearn haciendo ejercicios de tercero de la E.S.O de nivel fácil. (de los colores, los números y de ropa)

Día 16 de octubre estuve haciendo ejercicios de clicknlearn de $3^{\circ}$ de la ESO de países europeos.

Hoy es el último día de clase de inglés 16 de junio. Durante dos años hemos 
trabajado ingles tanto en los ordenadores como en la clase.

También compartimos con otros alumnos de otro colegio de Inglaterra nos mandábamos cartas de cómo era nuestro colegio y más cosas cotidianas que vivimos. Yo creo que me ha servido bastante todo lo que hemos hecho estos dos años he aprendido muchas cosas. Y lo mejor que hemos hecho es que lo hemos comparado $\mathrm{y}$ hemos visto las mejoras desde que empezamos hace dos años.

Es necesario aclarar que todos los participantes, salvo e6 que durante un periodo empleó la lengua extranjera para, posteriormente, pasar a utilizar su lengua materna, elaboraron sus diarios en español, pero, tal como se expuso en el apartado de metodología, se optó por ser flexible dado que el principal objetivo del diario era favorecer la reflexión y no la competencia lingüística en lengua inglesa.

Las actividades individuales y en grupo han resultado positivas puesto que los informantes han sido capaces de buscar, seleccionar y resumir la información requerida para, posteriormente, escribir un texto sencillo sobre temas corrientes o de interés personal, que se correspondería con un nivel A2 de acuerdo con los descriptores de la biografía lingüística del portafolio donde se pone de manifiesto que un aprendiz alcanza el nivel A2 cuando es capaz de «hacer descripciones y narraciones breves y/o extensas utilizando expresiones sencillas y frases cortas que han aprendido». Además han sido capaces de darlo a conocer a sus compañeros, aunque no fuese a modo de exposición sino a través de la lectura con lo que alcanzarían un nivel $\mathrm{A} 1^{+}-\mathrm{A} 2$ puesto que, tal como se refleja en el portafolio, «los discentes son capaces de hablar brevemente de experiencias y rutinas; describir cosas, lugares y personas» (http://www.oapee.es/documentum/ MECPRO/Web/weboapee/iniciativas/portfolio/portfolios-validados-esp/secundaria/castellano/pelbiografiadossiersecundaria.pdf?documentId $=0901 \mathrm{e} 72 \mathrm{~b} 800044 \mathrm{~b} 3$, visitada el $11 \mathrm{de}$ febrero de 2010). El extracto tomado del dossier de e7 pretende mostrar con datos objetivos lo expuesto con anterioridad, puesto que este informante es capaz de elaborar el texto que aquí se muestra partir de la lectura de varios textos similares en la página web: http://www.spanishkid.org y presentárselo a sus compañeros:

\section{e7's House}

Hi! My name is e7, I'm 16 years old, I lived ${ }^{2}$ in Oviedo (Spain). I lived with my father, mother and sister. I go to to school every day and I study Spanish and English. I have many friends in the school.

\section{My Family}

My grandmother's name is X and she's very adult, she lived with my grandfather, he's name is X. They live in Valladolid. My other grandfather live in Villaviciosa he's name is $\mathrm{X}$ he's a hard working person, and my other grandmother's name is $\mathrm{X}$, she's dead. My father also is hard-working person and he like cooking, Real Madrid football team and formula 1.My mother's name is X, she is a gossip and she like the order. My sister's name is $\mathrm{X}$, she has boyfriend, and studies in the university tourism.

\footnotetext{
${ }^{2}$ Se opta por poner en letra cursiva y no corregir los errores léxicos y gramaticales para que, así, se pueda comprobar el nivel de competencia lingüística en lengua inglesa del alumno.
} 
En lo que respecta al trabajo en grupo, se cumplieron los objetivos logrando mantener la atención del alumnado para poder responder a las preguntas propuestas por los autores del trabajo. Se trataba de preguntas sencillas que, previamente, habían sido revisadas por el profesor para comprobar que se ajustaban al nivel A1-A2 de comprensión oral (escuchar) según los descriptores del portafolio. Una vez corregidas las respuestas, se observa que son capaces de responder con sentido a lo que se les plantea con lo que alcanzan el nivel A2 puesto que son «capaces de captar el tema de las conversaciones y el sentido general de mensajes breves, claros y sencillos, relacionados con la vida cotidiana, comprender explicaciones breves de los compañeros, si tratan de temas conocidos y la pronunciación es clara y pausada» (http://www.oapee.es/inicio/iniciativas/ portfolio/niveles-de-competencia.html, visitada el 11 de febrero de 2010). Sin embargo, sus producciones escritas contienen errores gramaticales propios de alumnos con nivel de competencia $\mathrm{A} 1^{+}$-A2 en lengua inglesa, tal como se pone de manifiesto en las respuestas de e6 a los trabajos en grupo expuestos en el aula:

Answer the following questions:

- What kind of internet connections can you use with your PS3?

Cable or wifi

- When was Nintendo Corporation founded? 23th September 1889

- How many podiums has Pedrosa had in Valencia?

Three

- How old was Maniche when he started playing football professional? At 17 years old

- What does e8 have for breakfast at the weekend?

Coffe and biscuit.

Todos los participantes guardaban esta actividad corregida y evaluada por el profesor en sus dossiers, junto con una copia también corregida de sus trabajos y, posteriormente, se autoevaluaban en el portafolio en las destrezas de leer, escribir, hablar y escuchar para valorar la actividad de forma global. Tal como se refleja en el extracto del portafolio de e4 que se muestra a continuación, se observa que no se autoevalúa por encima del nivel A2 en la destreza de escribir, situación que se repite para el resto de destrezas, con lo que demuestra un proceso de reflexión adecuado y una evolución positiva en su aprendizaje como se comprueba a partir de las fechas en la que se llevó a cabo la autoevaluación:

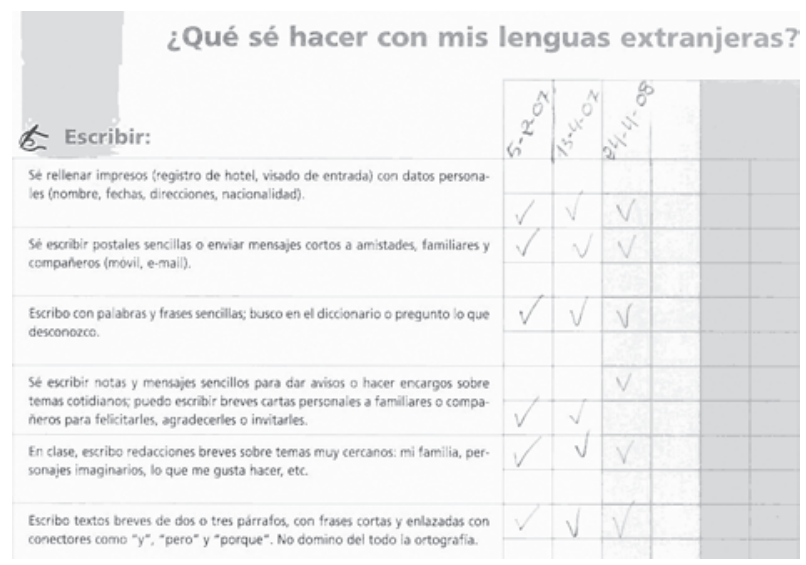


Esta situación se repite en el resto de los alumnos, salvo e5 que marca las casillas sin sentido hasta llegar a $\mathrm{C} 2$, con lo que muestra su nulo grado de reflexión y su escaso interés por la asignatura y la metodología aplicada, como así manifiesta en el cuestionario que se le administró al final del primer año de experimentación.

El e-mail tándem ha sido una experiencia positiva para los participantes, puesto que pudieron comunicarse con sus compañeros y ver las dificultades que sus parejas tándem encuentran a la hora de emplear el español en sus comunicaciones del mismo modo que les sucede a ellos a la hora de escribir en inglés. Como ellos mismos reconocen en sus diarios, esta experiencia les ha ayudado a reflexionar sobre su aprendizaje analizando los errores cometidos y lo que han aprendido de sus parejas, tal como se expuso al analizar los diarios al comienzo de este apartado. A continuación se incluye un extracto del diario de e3 en el que se incluyen su e-mail y el comentario sobre la experiencia del e-mail tándem:

\section{Hi X!}

How are you? We are very good. Your Spanish is good. Don't worry.

We are studing in X. Our school is a big building with a lot of class, we haven't got a sport centre, but we have got two gymnasiums, Science laboratory and library. Our school is beautiful. We like our school.

We wear uniform; it is dark blue skirt, jumper and white polo-necked.

Nos gusta llevar uniforme porque así no hace falta que pensemos qué ropa nos pondremos todos los días. Nuestro horario de clase es de ocho y media a dos y media, con un descanso de media hora en el que estamos con nuestras amigas.

Esperamos tú próxima carta. Greetings s3 y s4

Comentario de las cartas La verdad es que creo que ha sido una experiencia muy positiva en la que creo que he aprendido un montón de cosas más y sobre todo a expresarme un poco mejor. Hemos tenido algún que otro fallo que nos ha corregido

$\mathrm{X}$, pero la verdad es que pensé que tendríamos muchos más.

En lo que se refiere a las estrategias de aprendizaje los alumnos cumplimentaron un cuestionario sobre estrategias de comprensión lectora tomado de Palacios Martínez (2006: 165) con un resultado bastante negativo, puesto que no aplicaron ninguna de las estrategias dado que, debido a sus peculiares características, la lectura no es una de sus predilecciones. En lo que respecta a las actividades propuestas en el portafolio, los alumnos realizaron la sección Mis Lenguas para reflexionar sobre las lenguas en las que se comunican con los demás. Esta actividad resultó muy positiva porque fueron conscientes del uso de cada lengua de las que conocen (normalmente español, inglés y francés, salvo un caso que también conocía el catalán) en un contexto determinado, siendo el español la utilizada en todos los ámbitos y reduciendo el uso de las lenguas extranjeras al ámbito escolar, como se pone de manifiesto en la tabla completada por e10: 


\section{Mis lenguas}

¿En qué lenguas me comunico con los demás?

\begin{tabular}{|c|c|c|c|}
\hline ¿Qué lengua? & $\begin{array}{l}\text { ¿Dónde la uso? } \\
\text { En casa, en la escuela, } \\
\text { con los amigos, etc. }\end{array}$ & $\begin{array}{l}\text { ¿Qué hago? } \\
\text { Hablo, leo, escucho } \\
\text { música, viajo, etc. }\end{array}$ & $\begin{array}{l}\text { ¿Dónde la aprendí? } \\
\text { En casa, la escuela, } \\
\text { viajes, con amigos, etc. }\end{array}$ \\
\hline Español & $\begin{array}{l}\text { En todos los } \\
\text { sitios. }\end{array}$ & Comunicarme, & $\begin{array}{l}\text { En todos los } \\
\text { sitios }\end{array}$ \\
\hline $\begin{array}{l}\text { En cla } \\
\text { Francés }\end{array}$ & En clase & $\begin{array}{l}\text { Leía, escucha } \\
\text { Escribir, lé́a }\end{array}$ & $\begin{array}{l}\text { En el } \\
\text { colegio. }\end{array}$ \\
\hline Inglés & Enclase & $\begin{array}{l}\text { Leo, escucho, } \\
\text { leo, estudiar } \\
\text { y hablo un } \\
\text { poce. }\end{array}$ & En el colegio \\
\hline
\end{tabular}

Posteriormente, debieron dar forma a esta tabla con la actividad Mi abanico lingüístico, partiendo de un gráfico en forma de abanico en el que cada color representa una lengua y cada triángulo la edad desde la que el informante la emplea, todos los estudiantes elaboraron un texto con la información recogida en Mis lenguas utilizando el español y el inglés, como se refleja en el abanico de e2 que se muestra a continuación: 


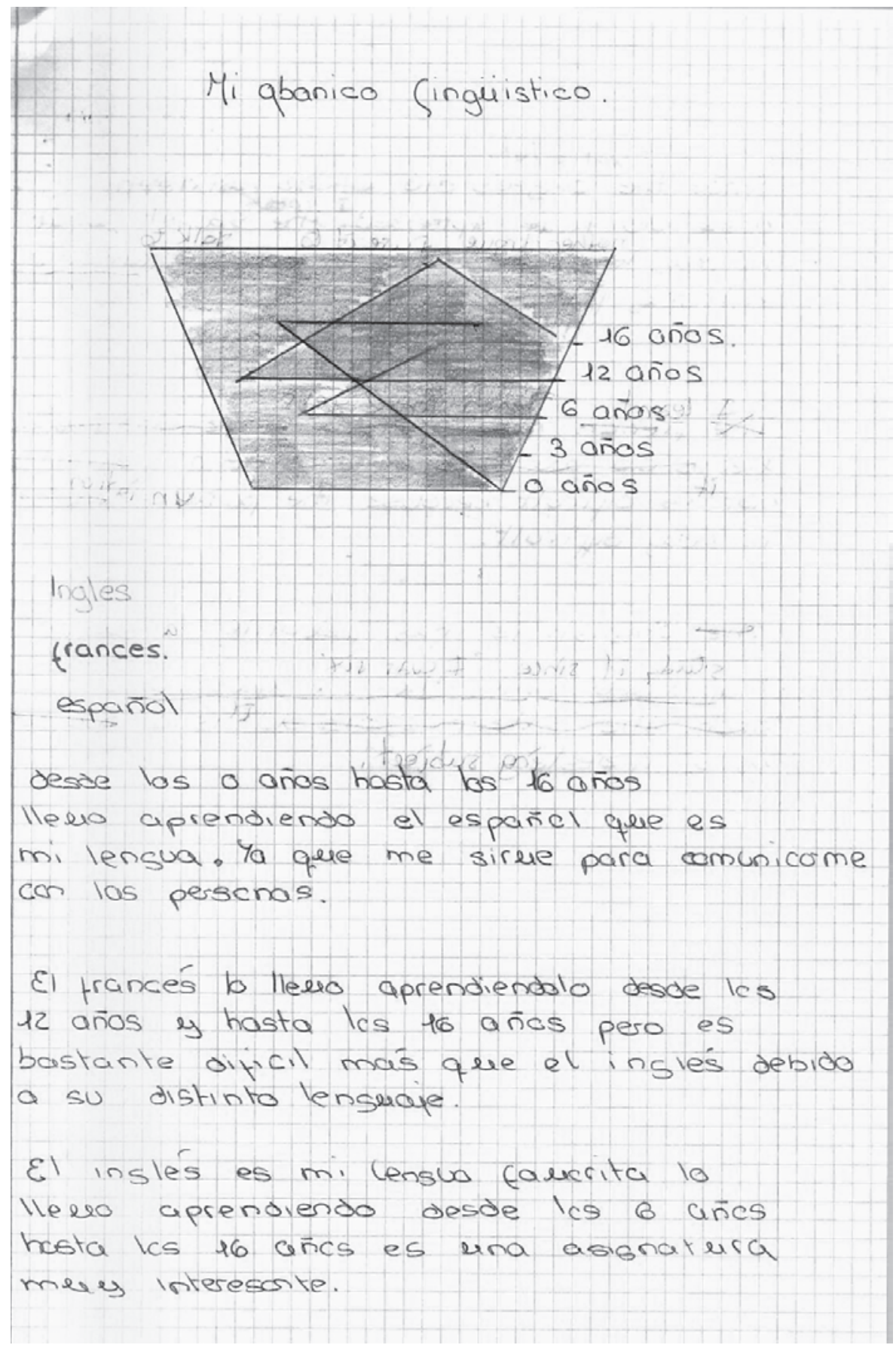




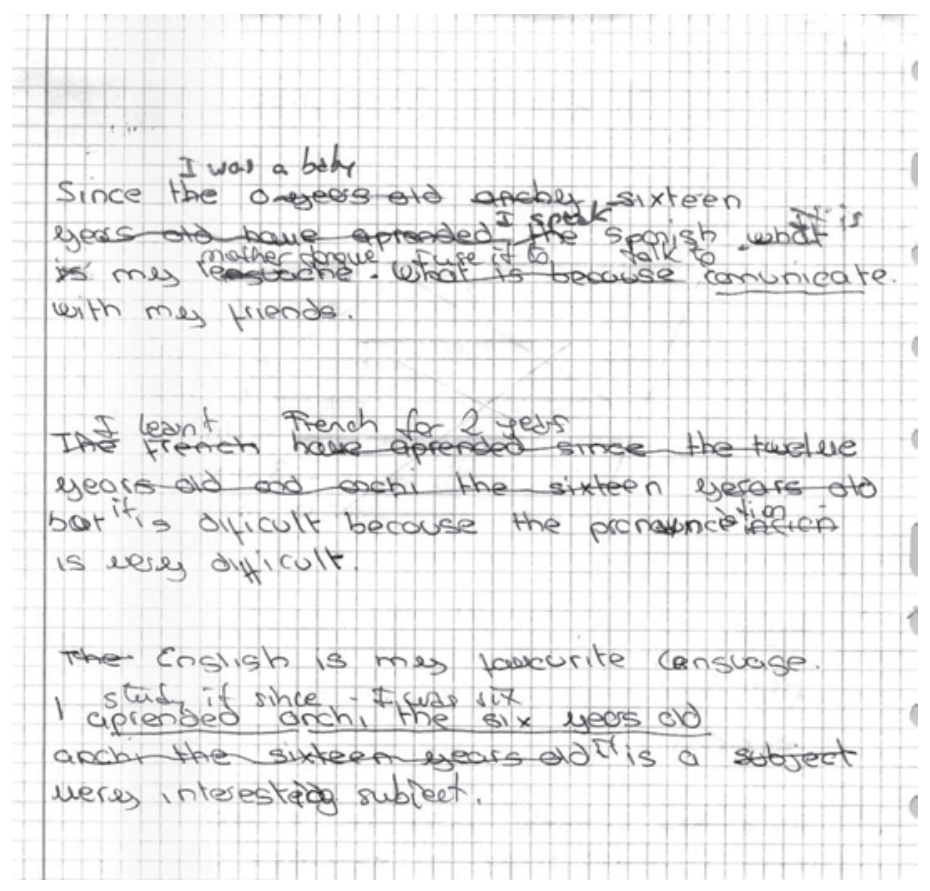

Por último, los cuestionarios administrados a los alumnos durante los dos años del estudio junto con las actividad Mi manera de aprender de la Biografía Lingüística han sido adecuados por un doble motivo. En primer lugar, gracias a los cuestionarios de autoevaluación los alumnos han reflexionado sobre su aprendizaje desde el comienzo del curso escolar 2006-2007 con el cuestionario de autoevaluación de la evaluación inicial y se han marcado metas que eran revisadas al final de cada trimestre, como se muestra en los ejemplos siguientes tomados de e6:

\section{AUTOEVALUACIÓN EVALUACIÓN INICIAL (Septiembre 2006)}

1. Piensas que has demostrado tus conocimientos....

De forma adecuada mejor de lo que esperabas $\mathrm{X}$ peor de lo que esperabas

2. Has sido capaz de mostrar tus conocimientos y capacidades de forma más adecuada en:

Compresión escrita (Reading)

$\mathrm{X}$ Expresión Escrita (Writing)

Compresión Oral (Listening)

X Gramática (Grammar)

3. El examen te resultó ¿fácil o difícil? ¿Por qué?

Me pareció normal porque son cosas que dimos pero no nos acordamos de ellas.

4. ¿Qué nota te pondrías? Justifica tu respuesta.

Un 5 porque vi aciertos y fallos por igual.

5. Medidas que vas a tomar para mejorar tu rendimiento en futuras pruebas 


\begin{tabular}{|c|c|c|c|}
\hline Reading & En el colegio & En casa & En otros lugares \\
\hline Writing & & $\begin{array}{c}\text { Repasar en casa } \\
\text { los textos en } \\
\text { inglés. }\end{array}$ & \\
\hline Listening & $\begin{array}{c}\text { Escuchar los textos más } \\
\text { atentamente. }\end{array}$ & $\begin{array}{c}\text { Escribir en casa } \\
\text { algún texto }\end{array}$ & \\
\hline Grammar & & $\begin{array}{c}\text { Mirarlo más } \\
\text { tiempo }\end{array}$ & \\
\hline
\end{tabular}

\section{AUTOEVALUACIÓN 2a EVALUACIÓN (Marzo 2007)}

1. Piensas que has demostrado tus conocimientos....

$\mathrm{X}$ De forma adecuada mejor de lo que esperabas peor de lo que esperabas

2. Has sido capaz de mostrar tus conocimientos y capacidades de forma más adecuada en:

$\mathrm{X}$ Comprensión escrita (Reading)

X Expresión Escrita (Writing)

$\mathrm{X}$ Comprensión y expresión oral (Listening, speaking and spoken interaction)

X Gramática (Grammar)

3. ¿Qué medidas has aplicado para lograr esa mejora tomando como referencia las que has sugerido en el cuestionario realizado tras la $1^{\circ}$ evaluación?

\begin{tabular}{|c|c|c|c|}
\hline & En el colegio & En casa & En otros lugares \\
\hline Reading & & Repasar encasa & \\
\hline Writing & & & $\begin{array}{c}\text { Escuchar música } \\
\text { por la calle. }\end{array}$ \\
\hline Listening & $\begin{array}{c}\text { Escuchar los textos más } \\
\text { atentamente. }\end{array}$ & & \\
\hline Grammar & & & \\
\hline
\end{tabular}

4. ¿Cómo te resultaron las pruebas realizadas a lo largo de la evaluación? Justifica tu respuesta.

Bien. Creo que no saqué mucha nota pero me salieron bien.

Los dos cuestionarios implementados al final del primer año sobre el portafolio y la metodología de aprendizaje seguida en el aula (Anexos I y II) fueron valorados muy positivamente por el alumnado participante, ya que únicamente dos informantes (e3 y e5) creen que usar el portafolio en el aula es una pérdida de tiempo mientras que todos los demás se manifiestan a favor de su empleo en la práctica docente porque: «sabes en lo que mejoras a lo largo del año» (e8) o «es importante hacerte responsable de tus actitudes académicas, así sabrás donde poner tu listón» (e9). En lo que respecta a la pregunta sobre el posible uso del PEL en otras asignaturas únicamente e3 y e5 no ven posibles aplicaciones en otras asignaturas, mientras que la mayoría lo ven útil para el aprendizaje de lenguas extranjeras y ely e9 lo ampliarían a cualquier lengua incluida la materna. Sin embargo, no son capaces de asociar el uso del PEL con la autonomía puesto que 
entienden autonomía como «mejora de las calificaciones» o «conceder más importancia a la asignatura» a pesar de que consideran importante responsabilizarse de su aprendizaje como reflejan en sus respuestas a la pregunta «Me gusta hacerme responsable de mi aprendizaje de lenguas como propone el PEL». Por último, consideran que han aprendido más que otros años y son capaces de autoevaluarse justificando su respuesta, tal como se pone de manifiesto en la respuesta de e4:

Si tuvieras que dar una nota para evaluar tu trayectoria académica durante este curso ¿qué nota te pondrías y por qué?

Debida a mi gran mejoría me pondría un 6 , pero teniendo en cuenta mi mal desenvoltura frente a un examen de gramática y sobre todo ante un listenig, creo que mi nota tendría que bajar un punto, por mucho que me cueste asumirlo. Y teniendo en cuenta que en inglés siendo una asignatura acumulativa, creo que mi nota tendría que ser la de los pasados trimestres.

Finalmente, la actividad Mi manera de aprender fue llevada a cabo al final del segundo año para que los alumnos aplicasen las estrategias que habían empleado durante

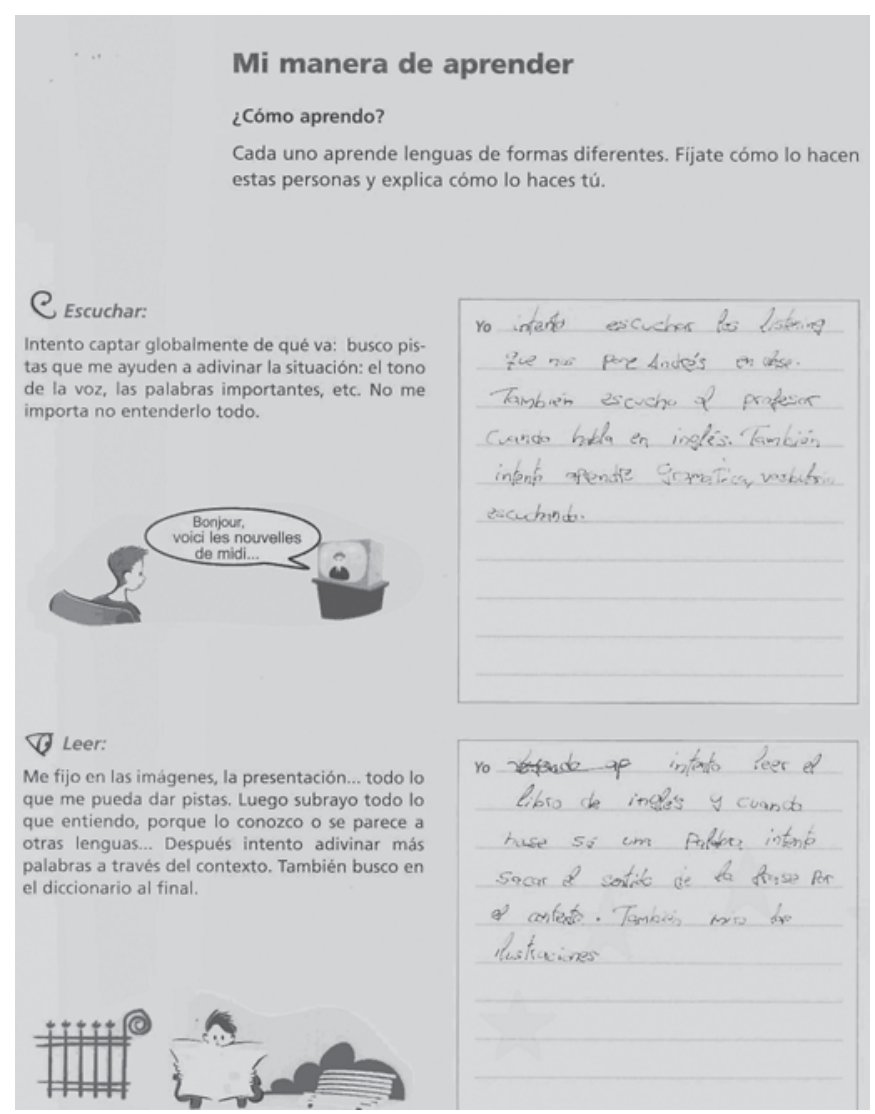
el estudio en lo referido a leer y escuchar siguiendo las sugerencias propuestas en el texto anexo. Tal como se muestra en el extracto del portafolio de e10, los estudiantes han sido capaces de enumerar las estrategias que utilizan con cada una de las destrezas, con lo que se pone de manifiesto la utilidad del uso del portafolio en el proceso de aprendizaje de una lengua extranjera en alumnos con dificultades de aprendizaje.

\section{Conclusiones}

Los resultados del presente estudio ponen de manifiesto que los informantes son más autónomos que al comienzo del mismo, puesto que son capaces de reflexionar sobre su proceso de aprendizaje sirvién- 
dose de sus diarios y de los descriptores del portafolio, al tiempo que consideran la experiencia como positiva para su formación y aprendizaje a lo largo de la vida. Esta reflexión coincide con el nivel de competencia lingüística que han alcanzado en lengua inglesa, tal como se ha mostrado a partir de las tareas individuales y grupales que han llevado a cabo durante los dos cursos académicos en los que se implementó el portafolio, puesto que, salvo un alumno, ninguno de los discentes considera que tiene un nivel superior a $\mathrm{A} 1^{+}-\mathrm{A} 2$.

El e-mail tándem les ha ayudado a conocer la realidad de otros aprendices de lenguas de su misma edad perteneciente a la cultura de la lengua meta con lo que han desarrollado su competencia comunicativa intercultural y mejorado su competencia escrita en lengua inglesa.

Por todas estas razones, se puede afirmar que el Portafolio Europeo de las Lenguas es una herramienta muy útil para facilitar la reflexión y el aprendizaje de la lengua meta en alumnos con dificultades de aprendizaje. Se podría argumentar que se trata de un grupo muy reducido de informantes como para poder extraer conclusiones a nivel general, por lo que sería conveniente en el futuro realizar un estudio empleando una metodología similar con un mayor número de informantes con dificultades de aprendizaje y contrastarlo también con alumnos sin problemas significativos de aprendizaje para comprobar si se produce una diferencia significativa entre el grado de reflexión que alcanzan los integrantes de ambos grupos. Así mismo, sería interesante llevar a cabo un estudio en el que el portafolio se utilizase no sólo en una única asignatura de lenguas sino en todas las materias que tengan que ver con las lenguas que se imparten en el centro e incluso en los colegios e institutos con Secciones Bilingües, se podría trabajar desde cualquier materia en la que se utilice el aprendizaje integrado de lenguas y contenidos en lengua extranjera (AICLE).

Por último, el Espacio Educativo de Educación Superior (EEES) es un marco adecuado para introducir el portafolio en las asignaturas de idioma moderno de los grados para ayudar a los alumnos a reflexionar sobre las estrategias de aprendizaje que emplean (por ejemplo para recordar el vocabulario que aprenden), como así se ha puesto en marcha por un grupo de profesores de la Universidad de La Rioja a través de un proyecto de innovación docente durante el segundo semestre del curso académico 2009-2010.

\section{REFERENCIAS BIBLIOGRÁFICAS}

Brammerts, H. y Calvert, M. (2003). «Learning by communicating in tandem», en T. Lewis y L. Walker (eds.), Autonomous Language Learning in Tandem. Sheffield: Academy Electronic Publications, 45-59.

Byram, M. (1997). Teaching and Assessing Intercultural Communicative Competence. Clevedon: Multilingual Matters.

Cassany, D, (ed.). (2006). El Portfolio Europeo de las Lenguas y sus aplicaciones en el aula. Madrid: MEC (Colección Aulas de Verano).

Council of Europe Education. (2009). Recommendation of the Committee of Ministers on the use of the Council of Europe's "Common European Framework of Reference for Languages» (CEFR) and the promotion of plurilingualism: CM/Rec(2008)7E and Explanatory 
Memorandum, disponible en: http://www.coe.int/t/dg4/linguistic/, visitada el 11 de febrero de 2010.

Dobson, A. (2006). "Algunos aspectos de las políticas del Consejo de Europa para la educación lingüística: El Marco Común Europeo de Referencia y el Portfolio Europeo de las Lenguas», en D. Cassany (ed.), El Portfolio Europeo de las Lenguas y sus aplicaciones en el aula. Madrid: Ministerio de Educación, 13-35.

Guía del Portfolio Europeo de las Lenguas para enseñanza secundaria. MECD, disponible en: http://www.oapee.es/documentum/MECPRO/Web/weboapee/iniciativas/portfolio/portfoliosvalidados-esp/secundaria/castellano/pelguiadidacticasecundaria.pdf?documentId= 0901e72b800044be, visitada el 11 de febrero de 2010.

Holec, H. (1981). Autonomy in Foreign Language Learning. Oxford: Pergamon.

Lewis, T. y Walker, L. (eds.). (2003). Autonomous language learning in tandem. Sheffield: Academy Electronic Publications.

Little, D. (2007). «Language learner autonomy: Some fundamental considerations revisited», en Innovation in Language Learning and Teaching 1 (1)): 14-29.

Little, D. y Perclová, R. (eds.). (2001). European Language Portfolio: Guide for Teachers and Teacher Trainers. Council of Europe: Strasbourg.

Little, D., Ridley, J. y Ushioda, E. (eds.). (2002). Towards Greater Learner Autonomy in the Foreign Language Classroom. Dublin: Authentik.

Palacios, I. M. (2006). «Aprendiendo a aprender en el aula de las lenguas extranjeras. Las estrategias de aprendizaje y su tratamiento en el aula», en D. Cassany (ed.). El Portfolio Europeo de las Lenguas y sus aplicaciones en el aula. Madrid: Ministerio de Educación, 129-170.

Marco Común Europeo de Referencia para las lenguas. (2001). Estrasburgo: Consejo de Europa, disponible en: http://cvc.cervantes.es/obref/marco/, visitada el 9 febrero de 2010.

Stickler, U. y Lewis, T. (2003). «Tandem learning and intercultural competence», en T. Lewis y L. Walker (eds.). Autonomous language learning in tandem. Sheffield: Academy Electronic Publications, 93-104.

Vygotsky, L. (1978). Mind in Society. The Development of Higher Psychological Processes. Cambridge, MA: Harvard University Press.

Wallace, M. J. (1998). Action Research for Language Teachers. Cambridge: C. U. P.

http://www.oapee.es/inicio/iniciativas/portfolio/niveles-de-competencia.html, visitada el 11 de febrero de 2010).

http:/www.oapee.es/documentum/MECPRO/Web/weboapee/iniciativas/portfolio/portfolios-validados$\mathrm{esp} / \mathrm{secundaria/castellano/memoriaexperimentacionsecundaria.pdf?documentId=}$ 0901e72b800044b2, visitada el 11 de febrero de 2010.

http:/www.oapee.es/documentum/MECPRO/Web/weboapee/iniciativas/portfolio/portfolios-validadosesp/secundaria/castellano/pelbiografiadossiersecundaria.pdf?documentId=0901e72b800044b3, visitada el 11 de febrero de 2010.

http://www.isabelperez.com, visitada el 11 de febrero de 2010.

http://clicknlearn.net, visitada el 11 de febrero de 2010.

http://www.englisch-hilfen.de, visitada el 11 de febrero de 2010.

http://spanishkid.org, visitada el 11 de febrero de 2010.

http://www.slf.ruhr-uni-bochum.de, visitada el 14 de febrero de 2010. 


\section{Anexo I \\ Answer the following questions in English or Spanish}

1. ¿Qué opinas de las clases de inglés de este curso?

2. ¿Cuál es tu opinión sobre el uso del Portfolio Europeo de las Lenguas en clase?

3. ¿Crees que se podría utilizar el PEL den otras materias? En caso afirmativo señala qué asignaturas serían adecuadas. Justifica tu respuesta.

4. ¿Qué actividades te gustaron más este curso? Justifica tu respuesta.

5. ¿Qué actividades te gustaron menos este curso? Justifica tu respuesta.

6. ¿Crees que eres un estudiante más autónomo que el curso pasado? Justifica tu respuesta.

7. ¿Qué sugerencias propondrías para el próximo curso en clase de inglés?

8. Si tuvieras que dar una nota para evaluar tu trayectoria académica durante este curso ¿qué nota te pondrías y por qué?

\section{Anexo II \\ Cuestionario Portfolio Europeo de las Lenguas}

1. El PEL me ayuda a mostrar lo que sé hacer en otras lenguas

2. Me gusta hacerme responsable de mi aprendizaje de lenguas como propone el PEL

3. Me parece útil ser yo mismo quien auto -evalúe mi progreso y domino de las lenguas

4. Mis profesores estuvieron de acuerdo con mi auto-evaluación

5. El tiempo dedicado al PEL en clase ha sido provechoso

6. Se debe animar a todos los alumnos a tener su PEL

7. Es importante que todos los europeos aprendan al menos dos lenguas aparte de la propia 